\title{
Kinetics of submicron oleic acid aerosols with ozone: A novel aerosol mass spectrometric technique
}

\author{
J. W. Morris and P. Davidovits \\ Chemistry Department, Merkert Chemistry Center, Boston College, Chestnut Hill, MA, USA
}

J. T. Jayne, J. L. Jimenez, ${ }^{1}$ Q. Shi, C. E. Kolb, and D. R. Worsnop

Center for Aerosol and Cloud Chemistry, Aerodyne Research Inc., Billerica, MA, USA

\author{
W. S. Barney ${ }^{2}$ and G. Cass ${ }^{3}$ \\ Department of Environmental Engineering, California Institute of Technology, Pasadena, CA, USA
}

Received 8 January 2002; revised 15 February 2002; accepted 15 February 2002; published 15 May 2002.

[1] The reaction kinetics of submicron oleic (9-octadecanoic (Z)-) acid aerosols with ozone was studied using a novel aerosol mass spectrometric technique. In the apparatus a flow of size-selected aerosols is introduced into a flow reactor where the particles are exposed to a known density of ozone for a controlled period of time. The aerosol flow is then directed into an aerosol mass spectrometer for particle size and composition analyses. Data from these studies were used to: (a) quantitatively model the size-dependent kinetics process, (b) determine the aerosol size change due to uptake of ozone, (c) assess reaction stoichiometry, and (d) obtain qualitative information about the volatility of the reaction products. The reactive uptake probability for ozone on oleic acid particles obtained from modeling is $1.6( \pm 0.2) \times 10^{-3}$ with an upper limit for the reactodiffusive length of $\sim 10 \mathrm{~nm}$. Atmospheric implications of the results are discussed. INDEX TERMS: 0365 Atmospheric Composition and Structure: Troposphere-composition and chemistry; 0317 Atmospheric Composition and Structure: Chemical kinetic and photochemical properties; 0345 Atmospheric Composition and Structure: Pollution - urban and regional (0305); 0305 Atmospheric Composition and Structure: Aerosols and particles $(0345,4801)$; 0394 Atmospheric Composition and Structure: Instruments and techniques

\section{Introduction}

[2] In this work we present a first-time study of organic aerosol kinetics using a novel aerosol mass spectrometric technique. For our first set of experiments illustrating the capabilities of the technique, we chose to study the kinetics of oleic acid $\left(\mathrm{C}_{18} \mathrm{H}_{34} \mathrm{O}_{2}\right)$ aerosols with ozone. In addition to kinetics information, data from these studies were used to determine the aerosol size change due to uptake of ozone, assess reaction stoichiometry, and obtain qualitative information about the volatility of the reaction products. The size dependence of the aerosol kinetics as discussed by Hanson and Lovejoy [1995] is clearly demonstrated.

\footnotetext{
${ }^{1}$ Now at Department of Environmental Engineering, California Institute of Technology, Pasadena, USA.

${ }^{2}$ Now at Arthur D. Little, Inc., Acorn Park, Cambridge USA.

${ }^{3}$ Deceased.
}

\section{Experimental}

[3] The aerosol kinetics apparatus is shown in Figure 1. Polydisperse aerosols, produced in a constant output atomizer (TSI Model 3076) from a solution of oleic acid in methanol, are entrained in a flow of dry filtered air at atmospheric pressure. Most of the methanol evaporates after passing through a silica gel diffusion dryer. (The amount of residual methanol in the particles is estimated from the particle mass spectra to be $2 \%$ by weight $=0.15$ mole fraction.) The oleic acid aerosols are then carried into a differential mobility analyzer (DMA - TSI Model 3071A). Within the DMA, particles are electrostatically classified by their mobility diameter. Most of the aerosols carry one, two or three charges. Therefore, three or more known sizes are pre-selected and emerge simultaneously from the DMA. The selected distribution can be limited to one, singly charged size by tuning the DMA to large particles (typically $>500 \mathrm{~nm}$ in diameter, at the high end of the initial size distribution).

[4] The aerosols are then introduced into a $2.2 \mathrm{~cm}$ diameter flow tube via a movable injector. Ozone is introduced into the flow tube upstream of the aerosol injector. The flow in the reaction zone is laminar $(\operatorname{Re} \sim 100)$ at one atmosphere total pressure. By matching the flow speeds $(\sim 8 \mathrm{~cm} / \mathrm{s})$ of the carrier gas and injector flows entering the reaction zone, the aerosols are isokinetically injected along the center axis of the flow tube. By translating the injector from 10 to $60 \mathrm{~cm}$, the aerosol-ozone interaction time can be varied from 1 to $7 \mathrm{~s}$. The aerosols are then sampled (again isokinetically) at $1.5 \mathrm{cc} / \mathrm{s}$ flow, through a $120 \mu \mathrm{m}$ orifice opening into the AMS. Isokinetic conditions are necessary for accurate determination of gas-particle interaction times. We note that due to the small diffusion coefficient of submicron aerosols at $1 \mathrm{~atm}$ $\left(D \sim 10^{-5} \mathrm{~cm}^{2} / \mathrm{s}\right)$, the aerosols are largely confined to the center axis of the flow tube, within a cross section defined by that of the injector ( $\sim 0.6 \mathrm{~cm}$ diameter $)$.

[5] Details of the aerosol mass spectrometer (AMS) are described in Jayne et al. [2000]. Here we will provide only an overview of the device. The AMS, shown in Figure 1, consists of three differentially pumped chambers. The sampled aerosols enter the first chamber through an aerodynamic focusing lens [Liu et al., 1995] that focuses the particles into a narrow beam $(\sim 1 / 2 \mathrm{~mm}$ diameter $)$. The pressures at the lens entrance and exit are about 2 torr and $10^{-3}$ torr respectively. The pressure difference accelerates the aerosols through the lens, imparting velocities as a function of aerosol aerodynamic diameter [Liu et al., 1995]. In the present experiments, velocities range between $150 \mathrm{~m} / \mathrm{s}$ and $90 \mathrm{~m} / \mathrm{s}$ for $150 \mathrm{~nm}$ and $1 \mu \mathrm{m}$ particles respectively. The pressures in the next two chambers are $10^{-5}$ torr and $10^{-7}$ torr respectively, such that each particle maintains its velocity as it traverses the chambers. In the third chamber the particles strike a resistively heated surface where the aerosols vaporize in 20 to 200 microseconds depending 


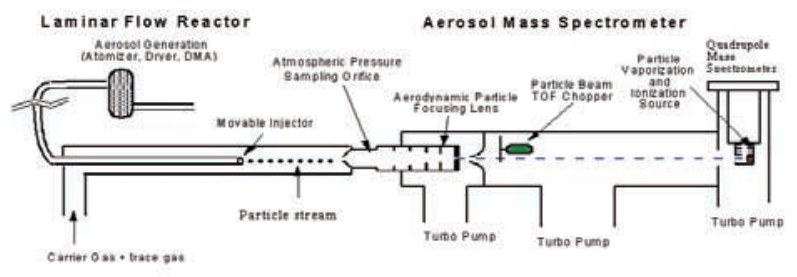

Figure 1. Aerosol kinetics apparatus.

on particle composition and heater temperature. The vapor plume is ionized by electron impact and the resulting ions are mass analyzed with a quadrupole mass spectrometer (UTI Model 100C). It has been experimentally verified that the mass spectrometer signal at the oleic acid parent peak is directly proportional to the amount of oleic acid in the particles (detection efficiency $\sim 10^{-7}$ ions counted per molecule).

[6] A two-slit chopper wheel positioned near the exit of the focusing lens intercepts the particle beam producing pulses of particles for time of flight (TOF) analysis. Typically the chopper rotates at $70 \mathrm{~Hz}$ with a $1.7 \%$ duty cycle. The TOF is determined by the elapsed time $\left(\sim 10^{-3} \mathrm{~s}\right)$ between the trigger pulse signaling the chopper opening and the ion pulse due to the vaporized aerosol. The ion signal is stored in a time-calibrated bin with $10 \mu$ s time steps. TOF can be converted to particle aerodynamic diameter, which for spherical particles is the product of the specific gravity and the geometric diameter.

[7] The AMS can be operated in either of two modes: In the TOF mode, signal proportional to the mass of the particles is monitored as a function of TOF or aerodynamic diameter for a given atomic mass unit (AMU). In the mass spectrum mode, the entire mass spectrum of the particles is displayed from 1 to 300 AMU. The TOF signal can be processed in both a single particle mode and in an integrated signal mode. In the integrated mode (used here) the quadrupole current is averaged over $10^{3}$ to $10^{4}$ particles.

\section{Results and Discussion}

[8] In Figure 2 we show mass spectra obtained from the vaporized particles of pure oleic acid (solid line) and oleic acid after exposure to ozone (dotted line). In this experimental run the diameter of the particles was approximately $600 \mathrm{~nm}$. The reacted particles were exposed to $\mathrm{O}_{3}$ at about $10^{-5}$ atm for 7 seconds. The pure oleic acid mass spectrum in Figure 2 compares well with the

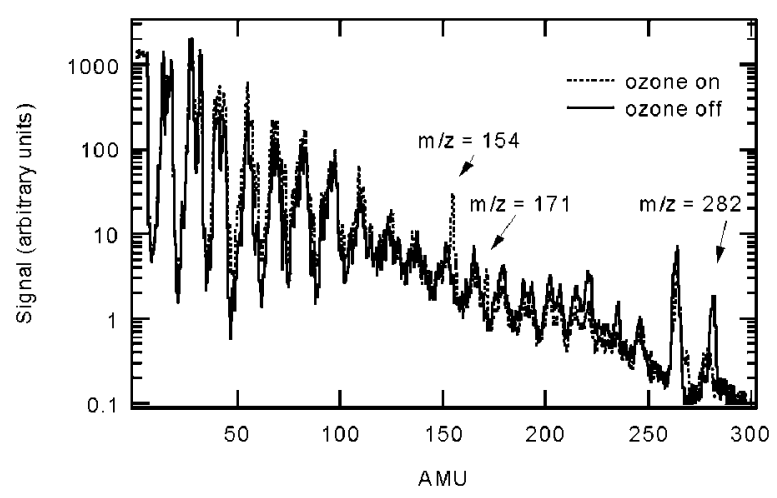

Figure 2. Particle mass spectrum for oleic acid aerosols with and without ozone.

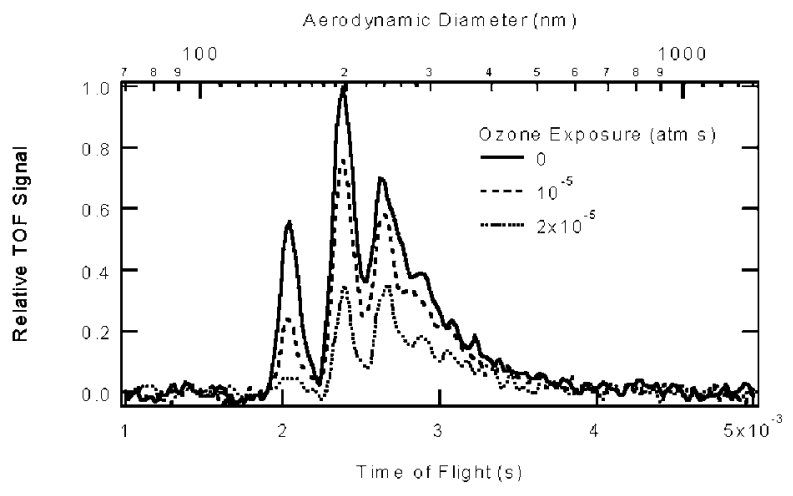

Figure 3. Time of flight (TOF) signal (mass 282) for oleic acid aerosol with and without ozone.

one in the NIST library [NIST, 2001]. The signal at mass 282 is the oleic acid parent peak. The decrease in this signal in the presence of ozone is clearly evident, as is the appearance of product peaks at masses 154 and 171 . We have chosen the oleic acid parent peak at mass 282 for our kinetic analysis.

[9] The reaction of ozone with oleic acid proceeds most likely via insertion of ozone to the double bond, forming an ozonide with pathways to several possible products. The observed peaks appearing with ozone exposure are likely due to product decomposition at the vaporizing heater or ionizer. The observed mass peaks are consistent with a recent $\mathrm{O}_{3}$ /oleic acid flowtube study of Moise and Rudich [2002] who detected heptanoic and azelaic acids in the collected liquid phase.

[10] In Figure 3 we show the TOF signals at mass 282 in the absence of ozone and at two ozone exposures $\left(P_{\mathrm{x}} t\right)$. The abscissa at the top designates the corresponding aerodynamic diameter of the particles. Three peaks are clearly resolved at 140,200, and $250 \mathrm{~nm}$ corresponding to singly, doubly and triply charged particles. Here, the width of the TOF signals is due mainly to three factors: DMA resolution, which for our flow conditions is typically $D / \Delta D \sim 15$, chopper transmission period $(\sim 0.1 \mathrm{~ms})$, and particle vaporization time [Jayne et al., 2000].

[11] Two features are evident in Figure 3. The signal at mass 282 decreases significantly with ozone exposure and the fractional loss is greater for smaller diameter particles. For example, at an exposure of $2 \times 10^{-5} \mathrm{~atm} \mathrm{~s}$, the fraction remaining for the 140 and $200 \mathrm{~nm}$ particles is 0.1 and 0.3 respectively.

[12] In Figure 4 the TOF signal for $600 \mathrm{~nm}$ oleic acid particles (aerodynamic diameter $\sim 550 \mathrm{~nm}$ ) is shown in the absence of ozone and at two exposures. This figure highlights two other features present in the TOF data: (a) An increase in particle size with ozone exposure is evident in the rightward shift in the leading edge of the TOF signal. This shift is simultaneous with the decrease in oleic acid signal due to species reaction. For the $4 \times 10^{-5} \mathrm{~atm}$ s ozone exposure, the observed increase in time of flight is $25 \mu$ s which for the $600 \mathrm{~nm}$ oleic acid particles corresponds to a fractional volume increase of $\Delta V / V_{0}=0.06\left(D / D_{0}=1.02\right)$. (b) Also in Figure 4, it is apparent that the signal width is decreasing with reaction extent. The full width at half max is $0.34,0.24$, and $0.18 \mathrm{~ms}$ for $1.0,0.4$, and 0.2 fraction remaining respectively. We suggest that the decrease in the TOF width is due to faster vaporization of the particle as it strikes the vaporizing heater. This decrease is consistent with increased volatility of the reaction products compared to unreacted oleic acid.

[13] The data shown in Figure 2 can be quantitatively analyzed to provide information about the stoichiometry of the reaction. The integrated ion signal of the mass spectrum from 0 to $300 \mathrm{amu}$ is proportional to the total aerosol mass (assuming detection effi- 


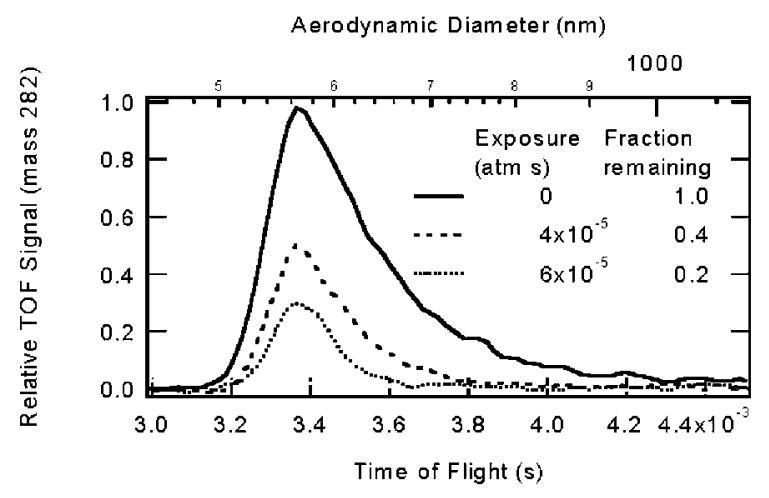

Figure 4. TOF signal (mass 282) for $600 \mathrm{~nm}$ diameter oleic acid aerosol without ozone and at two ozone exposures.

ciency is mass independent over the range of interest). Figure 5 plots the ratio of this total mass for ozone-on to ozone-off signals as a function of fraction oleic acid reacted, obtained from the signal ratios at the parent mass (282). The data points fall within $\sim 5 \%$ of the solid line which is the expected integrated mass ratio if a single $\mathrm{O}_{3}$ molecule reacted with each oleic acid molecule, and the products were retained in the particle. The dashed line assumes that two ozone molecules react per oleic acid molecule. As is evident, the former is consistent with the results. Similar information is obtained from the data in Figure 4 . by converting the change in TOF (measuring particle diameter) to a mass change.

\section{Kinetic Modeling}

[14] In our experiments, the gas-phase diffusion uptake coefficient, $\Gamma_{\text {diff }}[$ Hanson et al., 1996] ranges from 0.30 to 2.0. On the other hand, the reactive uptake coefficient $\Gamma_{\mathrm{rxn}}$ [Hanson and Lovejoy, 1995; Moise and Rudich, 2000] due to oleic acid-ozone reaction, is estimated to be on the order $10^{-3}$. The reactive uptake coefficient is therefore expected to govern the overall rate. A key parameter in the formulation of $\Gamma_{\mathrm{rxn}}$ is the ratio $l / a$, where $a$ is the particle radius and $l$ is the reacto-diffusive length, given by $l=(D /$ $\left.k_{2}[Y]\right)^{1 / 2}$. Here $D$ is the diffusion coefficient $\left(\mathrm{cm}^{2} \mathrm{~s}^{-1}\right)$ of ozone in oleic acid, $k_{2}$ is the second order rate constant for the reaction between ozone and oleic acid, and $[Y]$ is the concentration of oleic acid.

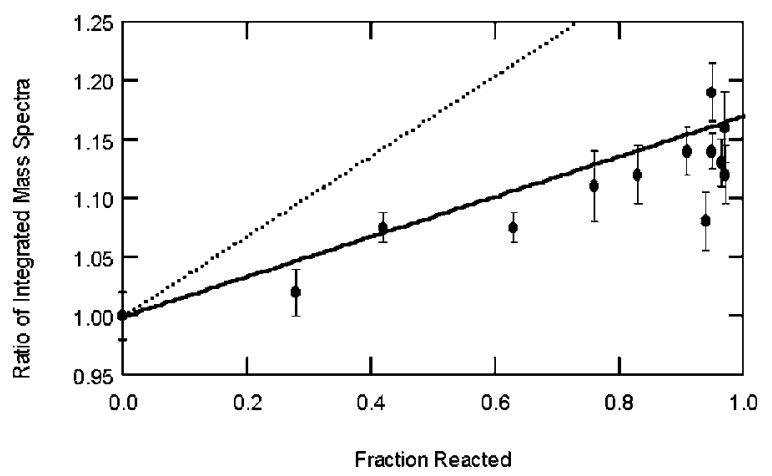

Figure 5. Ratio of the integrated mass spectra as a function of fraction reacted.
[15] The magnitude of $l$ can be estimated as follows. The initial concentration, $[Y]_{0}$ is $3.15 \mathrm{M}$. The parameters $D$ and $k_{2}$ have not been measured, however, they are expected to be on the order $D \sim 10^{-6} \mathrm{~cm}^{2} \mathrm{~s}^{-1}$ and $k_{2} \sim 10^{6} \mathrm{M}^{-1} \mathrm{~s}^{-1}$ [Rakovsky and Zaikov, 1998]. With these values, the reacto-diffusive length is estimated to be $l \sim 5 \mathrm{~nm}$. This is less than $10 \%$ of the radius for even the smallest particles studied here. In this limit, $\Gamma_{\mathrm{rxn}}$ is (see for example Hanson and Lovejoy [1995]):

$$
\Gamma_{r x n}=\frac{4 H R T}{\bar{c}} \sqrt{D k_{2}[Y]}
$$

Here, $H\left(\mathrm{M} \mathrm{atm}^{-1}\right)$ is the Henry's law coefficient for ozone in oleic acid, $R$ is the gas constant $\left(8.206 \times 10^{-2}\right.$ atm K $\left.{ }^{-1} \mathrm{M}^{-1}\right), T$ is the temperature, and $\bar{c}$ is the mean thermal speed of the impinging trace gas $\left(\mathrm{cm} \mathrm{s}^{-1}\right)$. The experimentally monitored quantity is the fraction of initial particle species $[Y] /[Y]_{0}$ remaining after a given exposure time at a known ozone pressure. With $\Gamma_{\text {rxn }}$ given by equation (1), this fraction can be expressed (to be shown in a forthcoming publication) as,

$$
\sqrt{\frac{[Y]}{[Y]_{0}}}=1-\frac{3 H \sqrt{D k_{2}}}{2 a \sqrt{[Y]_{0}}} P_{x} t
$$

where, $P_{x}$, is the partial pressure of ozone (atm). In equation (2) we have assumed $a(\mathrm{~cm})$ to be constant. Here the exposure, $P_{\mathrm{x}} t$, is the experimental variable. This expression indicates that the fractional consumption of oleic acid is more rapid in smaller particles, as is observed experimentally (Figure 3). Further, the model predicts that a plot of $\left([Y] /[Y]_{0}\right)^{1 / 2}$ as a function of the exposure yields straight lines with slopes inversely proportional to the particle radius.

[16] We note that as the $0.6 \mathrm{~cm}$ diameter particle stream enters the reaction zone, the particles do not immediately come into contact with the reactant trace gas (in this case $\mathrm{O}_{3}$ ). The trace gas must mix with the particle stream. If we assume that during some effective mixing time $\tau$, the aerosols are not in contact with the reactive gas, then the effective reaction time is $(t-\tau)$, which is to be substituted in equation (2).

$[17]$ In Figure 6 we show plots of $\left([Y] /[Y]_{0}\right)^{1 / 2}$ as a function of $P_{x}(t-\tau)$ for three particle diameters; $200 \mathrm{~nm}, 400 \mathrm{~nm}$, and $600 \mathrm{~nm}$. The straight lines in the figure show the global fit to the experimental data with two fitting parameters; $H\left(D k_{2}\right)^{1 / 2}$ and $\tau$. With $a$ and $[Y]_{0}$ given $\left([Y]_{0}=3.15 \mathrm{M}\right)$, best fit to the data is obtained with $H\left(D k_{2}\right)^{1 / 2}=0.33 \pm 0.03\left(\mathrm{M}^{1 / 2} \mathrm{~atm} \mathrm{~cm} \mathrm{~s}^{-1}\right)$ and $\tau=0.35 \mathrm{~s}$. The

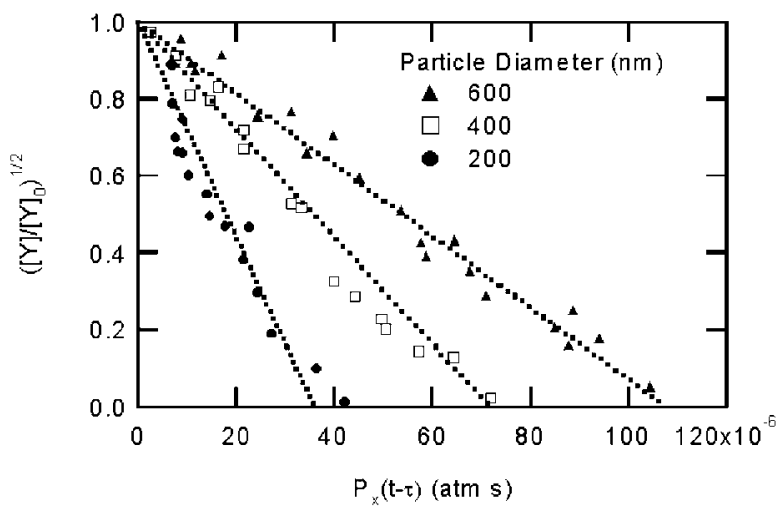

Figure 6. Oleic acid depletion in the form $\left([Y] /[Y]_{0}\right)^{1 / 2}$ as a function of exposure $P(t-\tau)$ for aerosol diameters 200, 400 and $600 \mathrm{~nm}$. The dashed lines are model fits to the data. 
effective mixing time of $0.35 \mathrm{~s}$ is consistent with the characteristic diffusion time into a $0.6 \mathrm{~cm}$ diameter stream $(\sim 0.5 \mathrm{~s})$. We note that the same value of the product $H(D k)^{1 / 2}$ fits the kinetics of the reaction over the full range of oleic acid decay. In the face of this result, one is lead to conclude that the parameters $H, D$, and $k$ either remain the same from reactant to product, or are changing in opposing directions such that the product remains nearly constant.

[18] As is evident, the observed oleic acid decay is in accord with the model, clearly exhibiting size dependent aerosol kinetics. With the fitted value of $H\left(D k_{2}\right)^{1 / 2}$, equation (1) yields the reactive uptake coefficient for ozone on oleic acid, $\Gamma_{\text {rxn }}=1.6( \pm 0.2) \times 10^{-3}$, in reasonable agreement with wetted wall experiments performed by Moise and Rudich [2002] using $\mathrm{O}_{3}$ and oleic acid and by de Gouw and Lovejoy [1998] using $\mathrm{O}_{3}$ and other liquid alkenes.

[19] In choosing the model to describe the present experiments we assumed that the reacto-diffusive length was much smaller than the particle radius $(1 \ll a)$. Using the more general formulation of the reactive process (allowing $1 \sim$ a) [Hanson and Lovejoy, 1995], the model is observed to fit the data only for $l<10 \mathrm{~nm}$. This defines an upper limit on the value $l=\left(D / k_{2}[Y]_{0}\right)^{1 / 2}$. With $[Y]_{0}$ known, it follows that $D / k_{2}<3.2 \times 10^{-12} \mathrm{~cm}^{2} \mathrm{M}$. An order of magnitude value for $H$ of ozone in oleic acid is estimated to be $10^{-1} \mathrm{M} \mathrm{atm}^{-1}$, using earlier approximated values for $D$ and $k_{2}$.

\subsection{Atmospheric implications}

[20] The value of $H\left(D k_{2}\right)^{1 / 2}$ obtained from data analysis implies an atmospheric half-life with respect to $\mathrm{O}_{3}$ reaction on the order of minutes for submicron aerosols of pure oleic acid in atmospheres polluted with $100 \mathrm{ppb} \mathrm{O}_{3}$. On the other hand, in the Los Angeles area, field measurements together with source flux estimates, imply an oleic acid atmospheric lifetime on the order of days [Rogge et al., 1991]. The longer atmospheric lifetime is likely due to the fact that oleic acid in atmospheric aerosols can be found in semi-solid, highly viscous mixtures such as with stearic and palmitic acids [Schauer et al., 1999]. The short reactodiffusive length $(l<10 \mathrm{~nm})$ confines the reaction to a thin shell near the surface of the aerosol, so that in such viscous particles the reaction is slowed down by diffusive transport of oleic acid to the surface.

[21] Acknowledgments. Funding was provided by NSF Grant Nos. ATM-99-05551 and CH-0089147, by DOE Grant No. DE-FG02-
98ER62581, and by the U.S.-Israel Binational Science Foundation Grant No. 1999134.

\section{References}

de Gouw, J. A., and E. R. Lovejoy, Reactive uptake of ozone by liquid organic compounds, Geophys. Res. Lett., 25, 931-934, 1998.

Hanson, D. R., and E. R. Lovejoy, The reaction of $\mathrm{ClNO}_{2}$ with submicrometer sulfuric acid aerosol, Science, 267, 1326-1328, 1995.

Hanson, D. R., A. R. Ravishankara, and E. R. Lovejoy, Reaction of $\mathrm{BrONO}_{2}$ with $\mathrm{H}_{2} \mathrm{O}$ on submicron sulfuric acid aerosol and the implications for the lower stratosphere, J. Geophys. Res., 101, 9063, 1996.

Jayne, J. T., D. Leard, Z. Zhang, P. Davidovits, C. E. Kolb, and D. R. Worsnop, Development of an aerosol mass spectrometer for size and composition analysis of submicron particles, Aerosol Science and Technology, 33, 49-70, 2000.

Liu, P., P. J. Ziemann, D. B. Kittelson, and P. H. McMurry, Generating particle beams of controlled dimensions and convergence: I. Theory of particle motion in aerodynamic lenses and nozzle expansions, Aerosol Sci. Technol., 22, 293-313, 1995a.

Moise, T., and Y. Rudich, Reactive uptake of ozone by proxies for organic aerosols: Surface versus bulk processes, J. Geophys. Res., 105, 14,66714,676, 2000 .

Moise, T., and Y. Rudich, Reactive uptake of ozone by aerosol-associated unsaturated fatty acids: Kinetics, mechanism and products, submitted to J. Phys. Chem., 2002.

NIST/EPA/NIH Mass Spectral Library, Search/Analysis Programs and Data for Microsoft Windows 95, 98, NT, 2000, January 2001.

Rakovsky, S., and G. Zaikov, in Kinetics of Ozone Reactions with Organic and Polymeric Compounds in Liquid Phase, Nova Science Publishers, 112,1998

Rogge, W. F., L. M. Hildemann, M. A. Mazurek, G. R. Cass, and B. R. T. Simoneit, Sources of fine organic aerosol. 1. Charbroilers and meat cooking operations, Environ. Sci. Technol., 25(6), 1112-1125, 1991.

Schauer, J. J., M. J. Kleeman, G. R. Cass, and B. R. T. Simoneit, Measurement of emissions from air pollution sources, $1, \mathrm{C}_{1}$ through $\mathrm{C}_{29}$ organic compounds from meat charbroiling, Environ. Sci. Technol., 33, 15661577,1999

J. W. Morris and P. Davidovits, Chemistry Department, Merkert Chemistry Center, Boston College, Chestnut Hill, MA 02467, USA.

J. T. Jayne, J. L. Jimenez, Q. Shi, C. E. Kolb, and D. R. Worsnop, Center for Aerosol and Cloud Chemistry, Aerodyne Research Inc., 45 Manning Road, Billerica, MA 01821, USA.

W. S. Barney and G. Cass, Department of Environmental Engineering, California Institute of Technology, Pasadena, CA 91125, USA. 